\title{
Non-syndromic megalencephaly and epilepsy: Our findings
}

Carmela Rita Massimino ${ }^{1}$, Silvia Marino ${ }^{2}$, Alessandro Giallongo ${ }^{1}$, Andrea Praticò ${ }^{1}$, Gloria Gangi ${ }^{1}$, Federica Filosco ${ }^{1}$, Claudia Oliva ${ }^{1}$, Giulia Lombardo $^{1}$ and Piero Pavone ${ }^{1}$

${ }^{1}$ Department of Clinical and Experimental Medicine, Section of Pediatrics and Child Neuropsychiatry, AUO Policlinico-Ove, University of Catania, Italy ${ }^{2}$ University-Hospital "Policlinico-Vittorio Emanuele," University of Catania, Italy

\begin{abstract}
Megalencephaly (MEG) and macrocephaly are defined as a head circumference measurements two standard deviations above the age-related mean. A distinction between megalencephaly and macrocephaly has been proposed despite the fact that these terms encompass individuals with a large head and, in some cases, with similar neurologic manifestations including intellective disability of various degree, epileptic seizures, and motor impairment. Nevertheless they differ widely in causal events, cerebral structural anomalies, approach in the work-up, treatment and prognosis for which a clinical distinction is justified. From July 2013 through July 2019, 10 children with non-syndromic MEG were selected (7 male, 3 female), and followed up at the Pediatric Unity of the University Hospital Policlinic-Vittorio Emanuele , Catania, Italy for pediatric and neuropediatric disorders; 5 of them (4 male, 1 female) have an abnormally large head, mild/moderate developmental delay, and seizures.
\end{abstract}

\section{Introduction}

Megalencephaly (MEG) and macrocephaly are defined as a head circumference measurements two standard deviations above the age-related mean [1,2]. A distinction between megalencephaly and macrocephaly has been proposed despite the fact that these terms encompass individuals with a large head and, in some cases, with similar neurologic manifestations including intellective disability of various degree, epileptic seizures, and motor impairment. Nevertheless they differ widely in causal events, cerebral structural anomalies, approach in the work-up, treatment and prognosis for which a clinical distinction is justified $[2,3]$.

MEG is a sign of several uncommon conditions and can be differentiated into three groups: idiopathic, metabolic, or anatomic. Anatomic MEG manifests with neurologic signs and may be isolated ("non-syndromic") or part of complex syndromes, such as Megalencephaly-Capillary Malformation-Polymicrogyria (MCAP) and macrocephaly-cutis marmorata telangiectasica (M-CMTC) [4-6].

Macrocephaly is referred to individuals in whom the brain enlargement is secondary to events inside the brain such as intracranial masses, abnormal ventricular dilatation, hydrocephalus ex vacuo, and increase of bone skeletal structures. On the other hand, megalencephaly refers to anomalous structural cerebral events such as ineffective molecular control of neuronal growth during the various stage of the brain development or to inborn errors of metabolism [2,4]. There are examples where the adverse events causing megalencephaly and macrocephaly may co-exist but semantic distinction among these conditions is suitable. In a previous article, our group seek to underline the clinical aspect of megalencephaly, emphasizing the main disorders that manifest with this anomaly in an attempt to properly categorize these disorders within the megalencephaly group [7].

We reviewed our patient with non-syndromic MEG, harboring minor dysmorphism, mild/moderate developmental delay and epileptic seizures as associated signs of the disorder: we enrolled 10 patients of MEG: five of them (unrelated patients) were affected by MEG and epilepsy.
From July 2013 through July 2019, 10 children with non-syndromic MEG were selected ( 7 male, 3 female), and followed up at the Pediatric Unity of the University Hospital Policlinic-Vittorio Emanuele, Catania, Italy for pediatric and neuropediatric disorders; 5 of them (4 male, 1 female) have an abnormally large head, mild/moderate developmental delay, and seizures.

\section{Editorial}

Among all the forms of MEG, the idiopathic form is the most frequent and is not associated with neurological manifestations, intellectual disabilities or other symptoms involving other parts of the body. In this disorder, the patient's head circumference increases gradually until an age of 18 months and then becomes more stable during the course of development. The children's parental OFC measurements were within normal ranges. This situation is opposite to what is typically reported in the literature for benign MEG (i.e., a large parental head is frequently reported) [5].

Brain MRI malformative anomalies were are uncommonly reported in children with MEG. This situation confirms the hypothesis of Berg and Dobyns [4], who maintain that genetic anomalies affecting brain development in the first steps of neuronal growth may be the cause of cerebral involvement; structural cerebral anomalies my not always be noticeable in brain imaging techniques.

This cohort of children exhibits a set of clinical signs consisting of an abnormally large head circumference (above the $97^{\text {th }}$ percentile),

*Correspondence to: Piero Pavone, University-Hospital "Policlinico-Vittorio Emanuele”, University of Catania, Italy, Via Plebiscito n. 667, 95100 Catania, Tel: 095 3781821; Fax: 0953782940; E-mail: ppavone@unict.it

Key words: epileptic seizures, megalencephaly, macrocephaly, large head, anatomic megalencephaly

Received: December 05, 2019; Accepted: December 10, 2019; Published: December 13, 2019 
a mild-to-moderate intellectual disability, epileptic seizures in 5 cases and, in some cases, minor dysmorphism especially facial. In the absence of signs suggestive of known syndromes, a diagnosis of anatomic, non-syndromic MEG was made. Nine of the ten patients did not shows structural abnormality of the brain, in the group with epilepsy four of the five patients failed to exhibit structural anomalies in their brain MRI. Only in one case we find a cerebral cortex dysplasia and micropolygyria. Among the children with epilepsy the EEG was abnormal and indicative of epilepsy diagnosis in all our patients; dysmorphisms were noted in three children; these conditions were not severe and mainly affected the face.

A differential diagnosis between idiopathic MEG and anatomic, non-syndromic MEG is not simple prior to the onset of clinical signs. Developmental delays, although usually mild and moderate, and dysmorphisms, if not severe, may be useful for a diagnosis.

We maintain that an abnormally large head deserves particular attention because this condition may hide relevant neurologic symptoms in the absence of evident brain anomalies. A large head in children with mild-to-moderate developmental delays and minor dysmorphisms may be a clue for subsequent epileptic seizures and a diagnosis of anatomic MEG.

\section{Acknowledgment}

We wish to thank the American Manuscript Editors (USA) for editing the manuscript.

\section{Statement of ethics}

The authors have no ethical conflicts to disclose.

\section{Disclosure statement}

The authors have no conflicts of interest to declare.

\section{References}

1. Sniderman A (2010) Abnormal head growth. Pediatr Rev 31: 382-384.

2. DeMyer W (1986) Megalencephaly: types, clinical syndromes, and management Pediatr Neurol 2: 321-328.

3. Winden KD, Yuskaitis CJ, Poduri A (2015) Megalencephaly and Macrocephaly. Semin Neurol 35: 277-287.

4. Berg AT, Dobyns WB (2015) Progress in autism and related disorders of brain development. Lancet Neurol 14: 1069-1170.

5. Mroske C, Rasmussen K, Shinde DN, Huether R, Powis Z, et al. (2015) Germline activating MTOR mutation arising through gonadal mosaicism in two brothers with megalencephaly and neurodevelopmental abnormalities. BMC Med Genet 16: 102.

6. Strauss KA, Puffenberger EG, Robinson DL, Morton DH (2003) Type I glutaric aciduria, part 1: Natural history of 77 patients. Am J Med Genet C Semin Med Genet 121: $38-52$

7. Pavone P, Praticò AD, Rizzo R, Corsello G, Ruggieri M, et al. (2017) A clinical review on megalencephaly: A large brain as a possible sign of cerebral impairment. Medicine (Baltimore) 96: e6814.

Copyright: (C2019 Massimino CR. This is an open-access article distributed under the terms of the Creative Commons Attribution License, which permits unrestricted use, distribution, and reproduction in any medium, provided the original author and source are credited. 